\title{
Pratiques phytosanitaires en agriculture périurbaine et contamination des denrées par les pesticides : cas des maraîchers de Port-Bouët (Abidjan)
}

Gains Kouakou KPAN KPAN"1, Lazare Brou YAO ${ }^{1}$, Chantal Assoh DIEMELEOU ${ }^{1}$, Roland Kossonou N'GUETTIA ${ }^{1}$, Sory Karim TRAORE ${ }^{1,2}$, Ardjouma DEMBELE ${ }^{1}$

1 Laboratoire National d'Appui au Développement Agricole (LANADA), Laboratoire Central d'Agrochimie et d'Ecotoxicologie (LCAE) 04 BP 612 Abidjan 04, Côte d'Ivoire.

2Laboratoire de Chimie Santé et Environnement, UFR des Sciences et Gestion de l'Environnement, Université Nangui Abrougoua, 02 BP 801 Abidjan 02, Côte d'Ivoire.

*Auteur correspondant; E-mail: oraclegains@gmail.com ; Tel. : (+225) 47663894

Publication date 31/07/2019, http://www.m.elewa.org/JAPS

1 RESUME

L'implication des pesticides dans l'agriculture urbaine nécessite l'examen des pratiques phytosanitaires adoptées par les maraîchers afin de s'assurer de la santé des maraîchers et des consommateurs. Ce travail a pour but d'examiner les pratiques phytosanitaires observées par les maraîchers de Port-Bouët et l'incidence de ces pratiques sur la qualité sanitaire des légumes. Pour ce faire, une enquête a été réalisée sur le mode d'utilisation des pesticides, puis des échantillons de laitue ont été collectés chez les maraîchers et les résidus de pesticides de ces échantillons ont été quantifiés par chromatographie en phase liquide à haute performance (HPLC-UV). Ces investigations ont montré que les pesticides utilisés étaient majoritairement des insecticides $(65,35 \%)$ suivis des insecticides/acaricides $(18,81$ $\%)$, puis des fongicides $(14,85 \%)$ et des insecticides/nématocides $(2,87 \%)$. Parmi ces pesticides, 39,6 \% n'étaient pas recommandés au maraîchage. La plupart des maraîchers $(86,84 \%)$ ne se protégeaient pas lors des applications phytosanitaires. La plus grande part des maraîchers $(80,26 \%)$ n'observaient pas les délais avant récolte. Au plan sanitaire, 93,33 $\%$ des échantillons de laitue n'étaient pas conformes aux normes du Codex alimentarius et de l'Union Européenne (Codex alimentarius, 2008; Codex alimentarius, 2011; Codex alimentarius, 2018 ; UE, 2018). En somme, les maraîchers de Port-Bouët n'observaient pas la bonne pratique phytosanitaire et cela avait pour conséquence la forte contamination de la laitue par les résidus de pesticides.

Mots clés : pesticides; maraîcher ; contamination ; Port-Bouët

\section{ABSTRACT}

The implication of pesticides in urban agriculture requires examination of phytosanitary practices adopted by gardeners to protect gardeners and consumers health. This work aims to examine the phytosanitary practices observed by gardeners who worked at Port-Bouët and the impact of these practices on vegetables quality. Thus, a survey was conducted on pesticides use, then lettuce samples were collected at production sites and pesticide residues were quantified by high performance liquid chromatography (HPLC-UV). These investigations showed that the pesticides used by gardners were insecticides $(65.35 \%)$, 
insecticides/acaricides (18.81\%), fungicides (14.85\%) and insecticides/ nematocides $(2.87 \%)$. A significant amont of pesticides (39.6\%) were not recommended for gardening. Most of the gardeners (86.84\%) did not protect themselves during pesticides application and $(80.26 \%)$ of these urban farmers did not observe the pre-harvest deadlines. Concerning vegetables quality, the pesticide residue content of $93.33 \%$ of lettuce samples were above maximum residue limits fixed by Codex Alimentarius and European Union (Codex alimentarius, 2008 ; Codex alimentarius, 2011; Codex alimentarius, 2018 ; UE, 2018). In conclusion, gardeners of Port-Bouët did not observe good phytosanitary practice ; as a result, lettuce was heavly contaminated by pesticides residues.

\section{2}

L'agriculture urbaine est une activité très présente dans les agglomérations africaines et participe activement à l'équilibre et à la dynamique de ces villes. Elle approvisionne le marché urbain en produits vivriers sur toute l'année (Jouve et Padilla, 2007; Olahan, 2010; Kouakou et al., 2010). Dans le district d'Abidjan, cette forme d'agriculture est pratiquée dans les bas-fonds, les marais et les berges lagunaires. Ainsi, chaque commune d'Abidjan, possède plus ou moins un site de production de cultures maraîchères (Adiko et al., 2006). Il s'agit d'une agriculture intensive qui utilise abondamment les pesticides pour accroître la production maraîchère (Dembélé, 2008; Dembélé, 2011). L'usage massif des pesticides peut avoir de sérieuses répercussions (teratogénicité, neurotoxicité, reprotoxicité et cancérogénicité) sur la santé des agriculteurs, des consommateurs et la qualité de l'environnement car la toxicité de ces intrants agricoles a été démontrée par plusieurs études toxicologiques et écotoxicologiques (Regelions, 2007; Meeker et al., 2008 ; Koutros et al., 2009; De Jaeger et al., 2012 ; Kpan Kpan et al., 2017; Kpan Kpan et al., 2018). En outre, des cas d'intoxication aigüe et chronique liés à l'exposition professionnelle et alimentaire aux produits agrochimiques ont été signalés un peu partout dans le monde chez les utilisateurs, les professionnels et les consommateurs (Sanborn et al., 2004 ; Multigner, 2005 ; De Jaeger et al., 2012). Face à la flambée des maladies auxquelles les pesticides sont potentiellement associés et l'apparition de cas cliniques d'intoxication aigüe et/ou chronique causés par les pesticides, l'utilisation et la gestion de ces substances sont devenues un sujet pluridisciplinaire très préoccupant. Ainsi, les pesticides sont étroitement surveillés par des organismes internationaux notamment l'Organisation des Nations Unies pour l'Alimentation et l'Agriculture (FAO) et l'Organisation Mondiale de la Santé (OMS) qui à travers leurs organes spécialisés, réglementent la synthèse, la vente et l'utilisation des pesticides puis fixent des valeurs toxicologiques de référence (VTR) des résidus de pesticides dans les denrées alimentaires et les matrices environnementales (OMS/FAO, 2010 ; FAO/OMS, 2018). Le respect des normes établies par ces organismes devrait conduire à l'usage et la gestion sécurisés des pesticides. Malheureusement, dans les pays émergeants ou en voie de développement, bon nombre d'utilisateurs des pesticides n'observent pas correctement les principes qui gouvernent l'utilisation sécurisée des produits agrochimiques (Doumbia et Kwadjo, 2009; Thiam et Sagna, 2009). Cette situation peut d'une part entraîner des intoxications chez les agriculteurs, les consommateurs et d'autre part la pollution de l'environnement en l'occurrence la contamination des eaux de surface, de la nappe phréatique et du réseau trophique (Roche et Ramade, 2011). Il est donc capital d'examiner les pratiques phytosanitaires impliquées dans les systèmes de production agricole des pays émergeants afin de remédier durablement aux failles qu'ils contiennent. En Côte d'Ivoire, le nombre d'études consacrées aux pesticides devient de plus en plus 
consistant. Certaines d'entre elles ont évalué le niveau de contamination des denrées alimentaires et des matrices environnementales par les résidus de pesticides (Traoré et al., 2008 ; Brou et al., 2016). D'autres études comme celles de Doumbia et Kwadjo (2009) et de Tano et al. (2011) se sont penchées sur l'utilisation et la gestion des pesticides par l'ensemble des maraîchers d'Abidjan et de Yamoussoukro. En dépit de ces études, l'incidence des pratiques

\section{MATERIEL ET METHODES}

3.1 Localisation des zones d'étude : La présente étude a concerné deux sites de production de plantes maraîchères localisés dans la commune de Port-Bouët. Il s'agit de la ceinture du 43ème Bataillon Infanterie Marine (43éme BIMa) et la zone de l'Agence pour la Sécurité de la Navigation Aérienne en Afrique et à Madagascar (ASECNA). Ces deux sites sont situés à l'est de la commune de PortBouët qui elle-même se trouve entre le $5^{\circ} 20^{\prime} 56^{\prime \prime}$ nord et le $4^{\circ} 00^{\prime} 42^{\prime \prime}$ ouest. La ceinture du 43è BIMa est la zone marécageuse qui entoure le camp phytosanitaires observées par les maraîchers sur le niveau de contamination des légumes que produisent ces agriculteurs n'a pas encore été abordée. La présente étude consacrée à la commune de Port-Bouët, entend examiner les pratiques phytosanitaires observées par les maraîchers de ladite commune et mesurer l'incidence de ces pratiques sur la qualité sanitaire de la laitue.

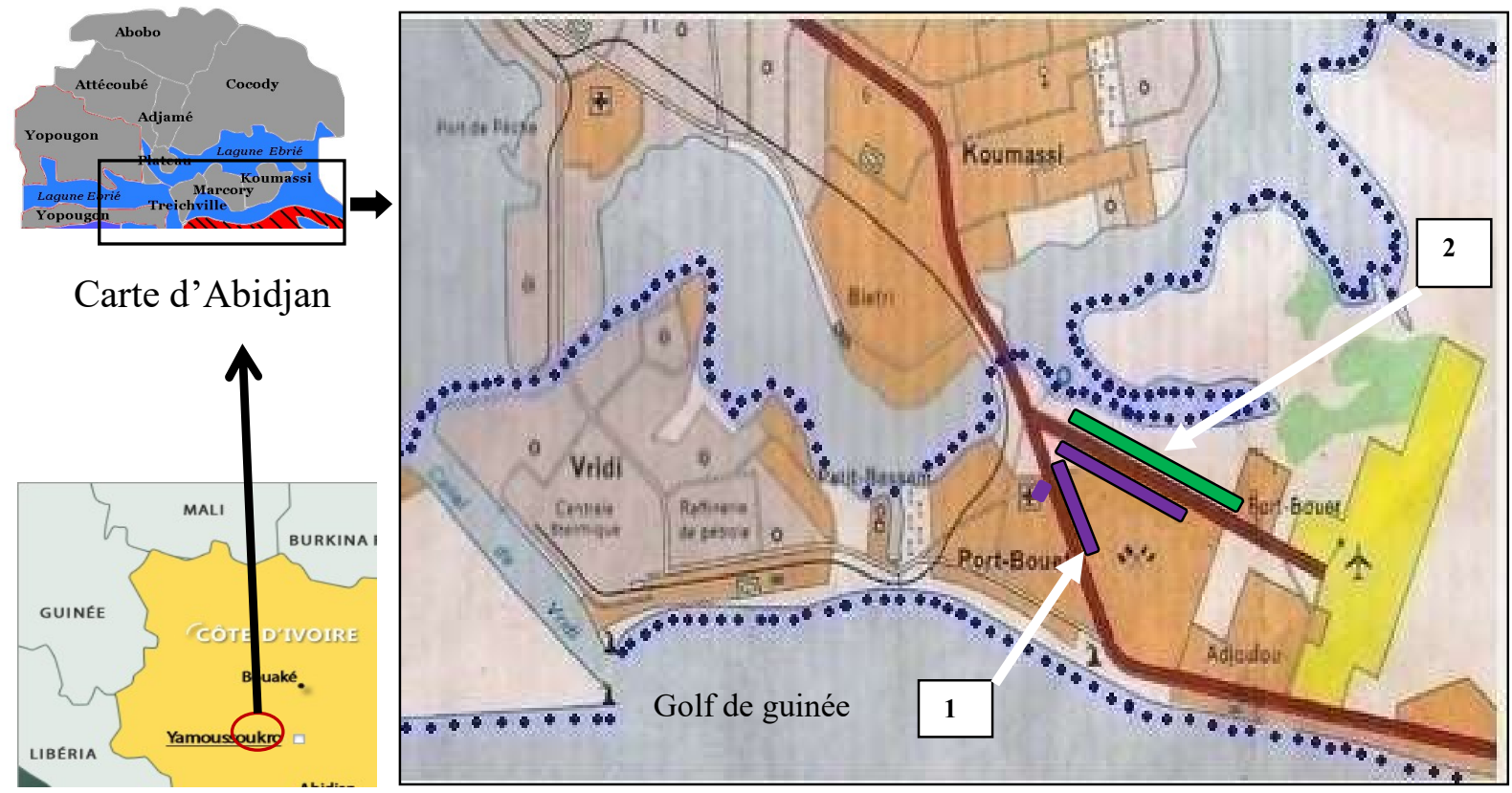

$1:$ zone du 43ème BIMa ; 2 : zone de l'ASECNA ; : limite de Port-Bouët

Figure 1 : Zones de production de la laitue à Port-Bouët (adaptée de Michelin, 1998)Echelle : 1/100000

3.2 Caractérisation des maraîchers : Pour la caractérisation des maraîchers, une prospection des deux sites de production maraîchère et une enquête militaire français; une zone constamment humide et favorable aux cultures maraîchères en toute saison. Quant à la zone de l'ASECNA, elle s'étend depuis ladite agence jusqu'au village d'Adjahui Namoué et à la lagune Ebrié (figure 1). Cette terre est parsemée de points d'eau et est par conséquent favorable au développement des cultures maraîchères. Les précipitations mensuelles varient entre $26 \mathrm{~mm}$ en janvier et $610 \mathrm{~mm}$ en juin et la température y est quasi constante(environ 27 degrés Celsius). 
d'identifier les différents types de pesticides utilisés et de vérifier le respect de l'adéquation entre les produits phytosanitaires et les cultures. Cette opération a consisté d'une part à collecter les différents emballages des spécialités agropharmaceutiques de la ceinture du 43ème BIMa et du secteur de l'ASECNA. Ces emballages ont été minutieusement scrutés afin de recueillir toutes les informations utiles. Ainsi, pour chaque produits agrochimique, les informations recherchées étaient le nom commercial du produit, la nature des matières actives, le dosage de la matière active, la dose usuelle, la nature de la culture ou des cultures à laquelle ou auxquelles est initialement destinée la formulation, le numéro d'autorisation de la mise sur le marché ou le numéro d'homologation, le nom du fabricant ou du distributeur et le délai avant récolte. Par ailleurs, cette prospection a permis d'examiner les points clés de l'application des pesticides notamment le port d'équipements de protection individuelle, les opérations de calibrage, le calcul des doses adéquates et la procédure de préparation des bouillies.

3.4 Interview des maraîchers: Dans le but d'une caractérisation des maraîchers par leurs modes d'utilisation et de gestion des produits agropharmaceutiques, une interview des maraîchers a été réalisée sur chaque site d'étude. Durant cette opération, un accent a été mis sur le nom du site, le sexe, l'âge, le niveau d'instruction, la formation au maraîchage, la protection de l'applicateur au cours $\mathrm{du}$ traitement, les malaises consécutifs à l'application des pesticides, les noms commerciaux des produits utilisés et le délai avant récolte observé par le maraîcher.

3.5 Teneur en résidus de pesticides de la laitue : Pour mesurer l'impact de l'utilisation des produits phytosanitaires sur les fruits et légumes produits par les maraîchers de Port-Bouët, des échantillons de laitue de $200 \mathrm{~g}$ chacun, pris de façon aléatoire, ont été analysés par chromatographie en phase liquide à haute performance (HPLC). Ces analyses ont été réalisées au Laboratoire Central d'Agrochimie et d'Ecotoxicologie. L'extraction et la purification se sont faites suivant les recommandations de Tekel et Hatrfk (1996) et Ambrus et al. (1981). Pour chaque échantillon, $50 \mathrm{~g}$ de broyat ont été prélevés puis $50 \mathrm{ml}$ d'eau bidistillée et $100 \mathrm{ml}$ d'acétone y ont été ajoutés. Ce mélange a été homogénéisé pendant 3 minutes à l'aide d'un mixeur Ultra Trax (OMNI International, USA). La mixture obtenue a subi une filtration sur du papier Whatmann contenant de la laine de verre. Pour séparer la phase aqueuse de la phase organique, le filtrat a été transféré dans une ampoule à décanter, puis $20 \mathrm{~g}$ de $\mathrm{NaCl}$ (Scharlau, Espagne) y ont été ajoutés et le mélange a été secoué vigoureusement. Après une brève phase de repos, le surnageant a été recueilli puis son volume a été réduit à $10 \mathrm{ml}$ à l'aide d'un évaporateur rotatif (BUCHI, Suisse). Pour purifier chaque extrait, une colonne en verre contenant du fluorisil activé (Acros Organics, USA) a été conditionnée avec $5 \mathrm{ml}$ d'acétone (Acros Organics, USA) puis les $10 \mathrm{ml}$ d'extrait ont été purifiés sur cette colonne. L'extrait purifié a été asséché à l'aide d'un évaporateur rotatif (BUCHI, Suisse). Les résidus de pesticides ont été récupérés avec $5 \mathrm{ml}$ d'hexane (ChemLab, Belgique) puis transvasés dans des vials pour la quantification des pesticides. La détection des pesticides a été réalisée à l'aide d'une chaîne de HPLC (SHIMADZU, Japon) composée d'un échantillonneur SIL-20A, d'une pompe LC-20AT, d'un réservoir TRAY, d'un dégazeur DGU-20A5, d'un four de type CTO-20A $\left(40^{\circ} \mathrm{C}\right)$ et d'un détecteur UV/VIS SPD-20A. L'élution a été réalisée en mode isocratic avec un débit de $0,5 \mathrm{ml} / \mathrm{min}$ pour tous les pesticides. Les autres paramètres analytiques sont donnés par le tableau 1.

Tableau 1: Paramètres analytiques de la détermination des résidus de pesticides

\begin{tabular}{|c|c|c|c|c|c|}
\hline \multirow[b]{2}{*}{ Matière active } & \multirow{2}{*}{$\begin{array}{c}\text { Longueur } \\
\text { d'onde } \\
\text { (nm) }\end{array}$} & \multicolumn{2}{|c|}{ Phase mobile } & \multirow[b]{2}{*}{ Colonne } & \multirow{2}{*}{$\begin{array}{c}\text { Volume } \\
\text { d'injection } \\
(\mu 1)\end{array}$} \\
\hline & & $\begin{array}{l}\text { Eau } \\
(\%)\end{array}$ & $\begin{array}{c}\text { Acétonitrile } \\
(\%)\end{array}$ & & \\
\hline Chlorothalonil & 271 & 20 & 80 & Nucléosil 5C18 & 10 \\
\hline Manèbe & 254 & 10 & 90 & Nucléosil 5C18 & 10 \\
\hline Chlorpyrifos & 254 & 10 & 90 & Nucléosil 5C18 & 10 \\
\hline Carbendazime & 210 & 30 & 70 & Nucléosil 5C18 & 10 \\
\hline Cyperméthrine & 230 & 10 & 90 & Nucléosil 5C18 & 10 \\
\hline Lambdacyhalothrine & 266 & 20 & 80 & Nucléosil 5C18 & 20 \\
\hline
\end{tabular}


Un étalonnage a été effectué à partir des standards purs de pesticide certifiés fournis par Dr Ehrenstorfer GmbH (Allemagne). Les surfaces des pics (standards et échantillons) ont permis de quantifier les différentes matières actives présentes dans les échantillons de laitue. Les différentes teneurs de pesticides ont été calculées à partir de la formule ci-dessous :

$$
C_{p}=\frac{S_{c} \times C_{e} \times V_{2} \times V_{f} \times F}{S_{e} \times M_{e} \times V_{1}}
$$

où $\mathrm{C}_{\mathrm{p}}$ : concentration de la matière active $(\mathrm{mg} / \mathrm{Kg})$; $S_{c}$ : surface du pic de l'échantillon; $S_{e}$ : surface du pic

\section{RÉSULTATS}

4.1 Caractérisation sociale des maraîchers : Au total, 76 maraîchers ont été interrogés sur l'ensemble des deux sites. Cet effectif de maraîchers, reparti entre les deux sites d'étude donnait 36 maraîchers pour la "ceinture » du 43è BIMa et quarante maraîchers pour la zone de l'ASECNA. du standard; $\mathrm{C}_{\mathrm{e}}$ : concentration du standard (mg /L); $\mathrm{V}_{1}$ : volume total d'extrait $(\mathrm{L}) ; \mathrm{V}_{2}$ : volume d'extrait purifié $(\mathrm{L}) ; \mathrm{V}_{\mathrm{f}}$ : volume final $(\mathrm{L}) ; \mathrm{M}_{\mathrm{e}}$ : masse de l'échantillon $(\mathrm{kg}) ; \mathrm{F}$ : facteur de dilution.

Les quantités de pesticide retrouvées dans les échantillons de laitue ont été comparées aux limites maximales de résidus (LMR) fixées par le Codex alimentarius ou par l'Union Européenne (UE) afin de déterminer l'acceptabilité de ces échantillons.

3.6Traitement statistique: Les statistiques descriptives et les histogrammes ont été réalisés à l'aide du tableur EXCEL intégré dans Microsoft Office 2013.

4.2 Répartition des maraîchers selon le sexe : La production des plantes légumières (labour, application des pesticides, arrosage, sarclage) était majoritairement assurée par les hommes. Ces derniers représentaient 98,68\% de l'effectif des maraîchers contre 1,32\% de femmes (figure 2).

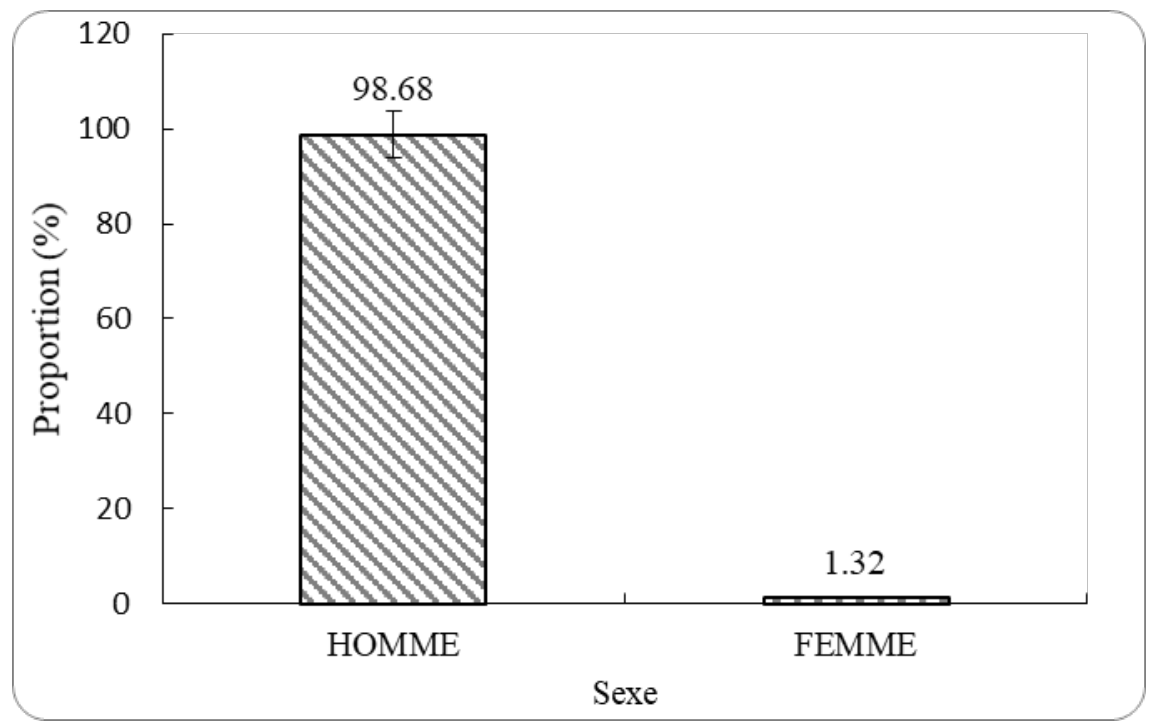

Figure 2 : Histogramme de la répartition des maraîchers en fonction du sexe

Les femmes rencontrées sur le terrain étaient les épouses et les filles des maraîchers ou des commerçantes. Elles assuraient essentiellement la commercialisation des produits maraîchers qu'elles livraient soit aux vendeuses du marché, soit aux acheteurs bord champ.
4.3 Niveau d'instruction des maraîchers : L'étude du niveau d'instruction des maraîchers a montré que le taux de scolarisation des maraîchers était faible. Il était de 17,11\% contre 82,89\% de non scolarisés. Parmi les scolarisés, 15,79\% avaient un niveau d'études $\leq$ Certificat d'Etude Primaire Elémentaire (CEPE) et $1,32 \%$ avaient un niveau 
d'études compris entre le CEPE et le baccalauréat (figure 3).

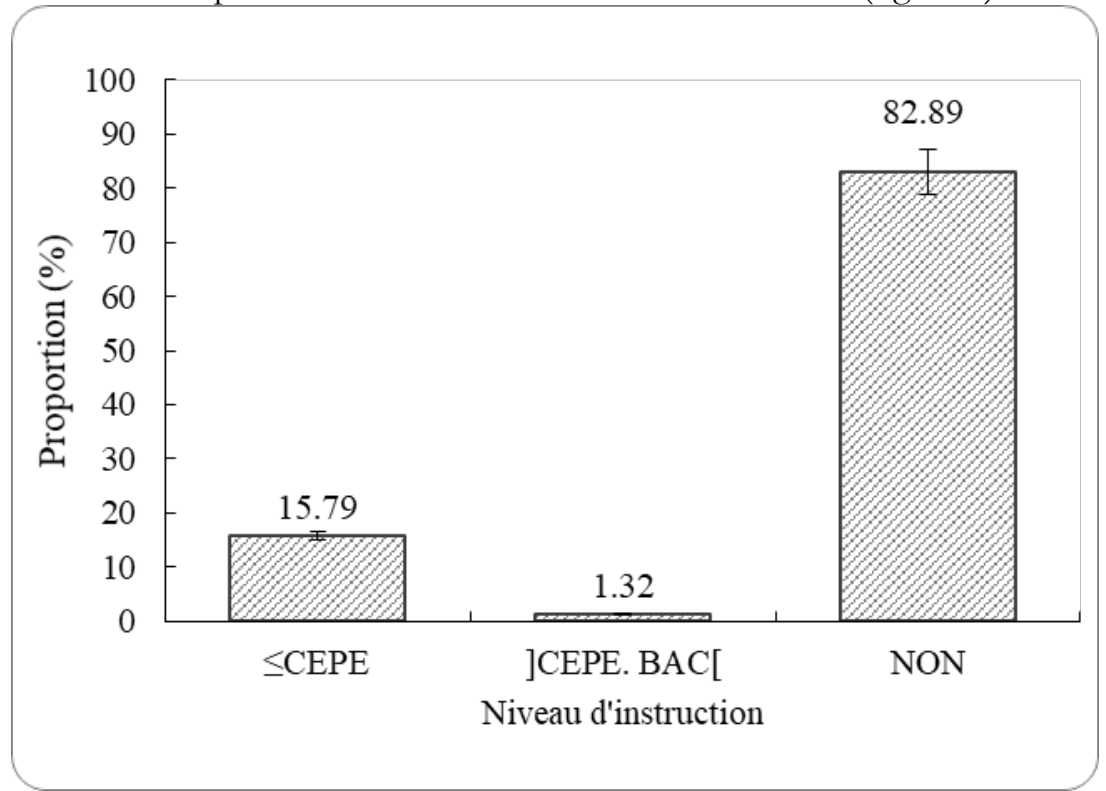

Figure 3 : Histogramme de la répartition des maraîchers en fonction le niveau d'instruction

4.4 Formation au maraîchage : La formation dont il a été question était celle que les maraîchers avaient obtenue de la part de structures de formation agréées telles que l'Agence Nationale d'Appui au Développement Rural (ANADER) et les Organisations Non Gouvernementales (ONG) agréées. A cet effet, 94,74 \% des maraîchers avaient affirmé n'avoir reçu aucune formation avant de s'adonner au maraîchage. Ils avaient appris ce métier sur le tas, par le canal de leurs prédécesseurs. Seulement 5,26\% des maraîchers ont déclaré avoir été formés par des structures agréées (figure 4).

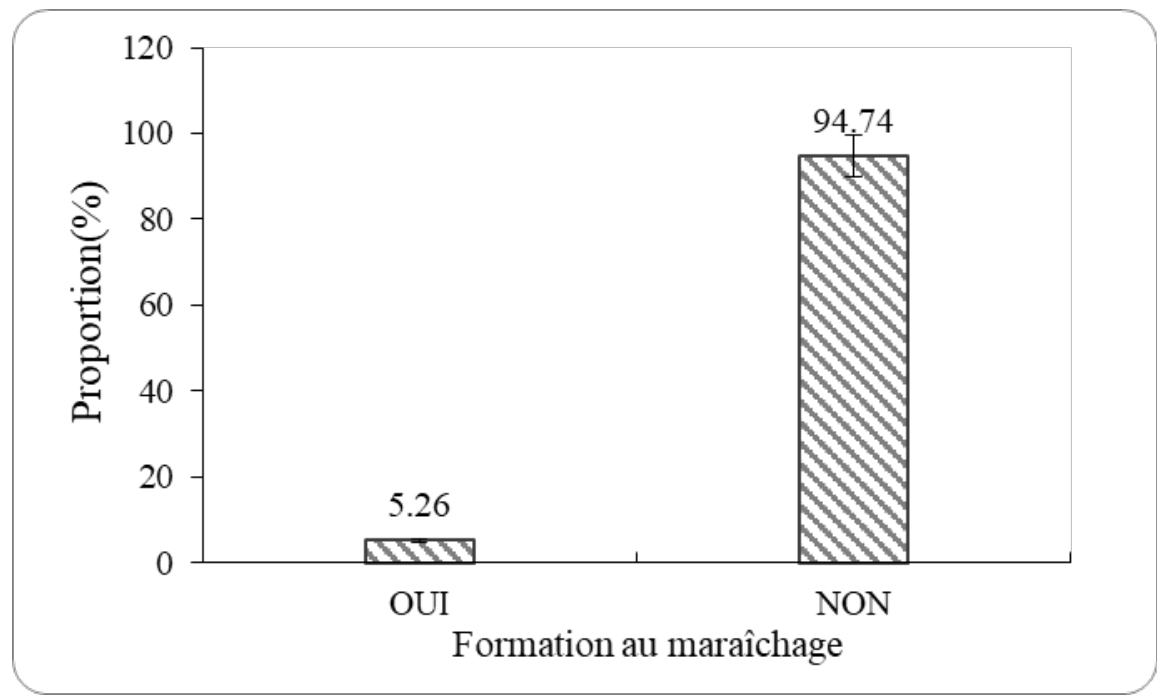

OUI : formé ; NON : non formé

Figure 4 : Histogramme de la répartition des maraîchers en fonction de la formation au maraîchage

Sur la question de l'assistance des maraîchers dans leur travail par les structures agricoles étatiques ou des ONG, certains maraîchers avaient affirmé qu'ils n'avaient reçu aucune assistance. D'autres par contre, ont déclaré avoir reçu de l'assistance de la part de l'ANADER, assistance qui a cessé il y avait de cela dix (10) ans. 
4.5 Pratiques Phytosanitaires: La collecte d'emballage au niveau des deux sites d'études (43è BIMa et ASECNA) a permis d'obtenir 101 emballages dont 30 pour le site du 43è BIMa et 71 pour le site de l'ASECNA. Les figures 5 et 6 présentent les emballages du Cypercal 50 EC et de Starter 350 EC découverts sur le site du 43è BIMa.
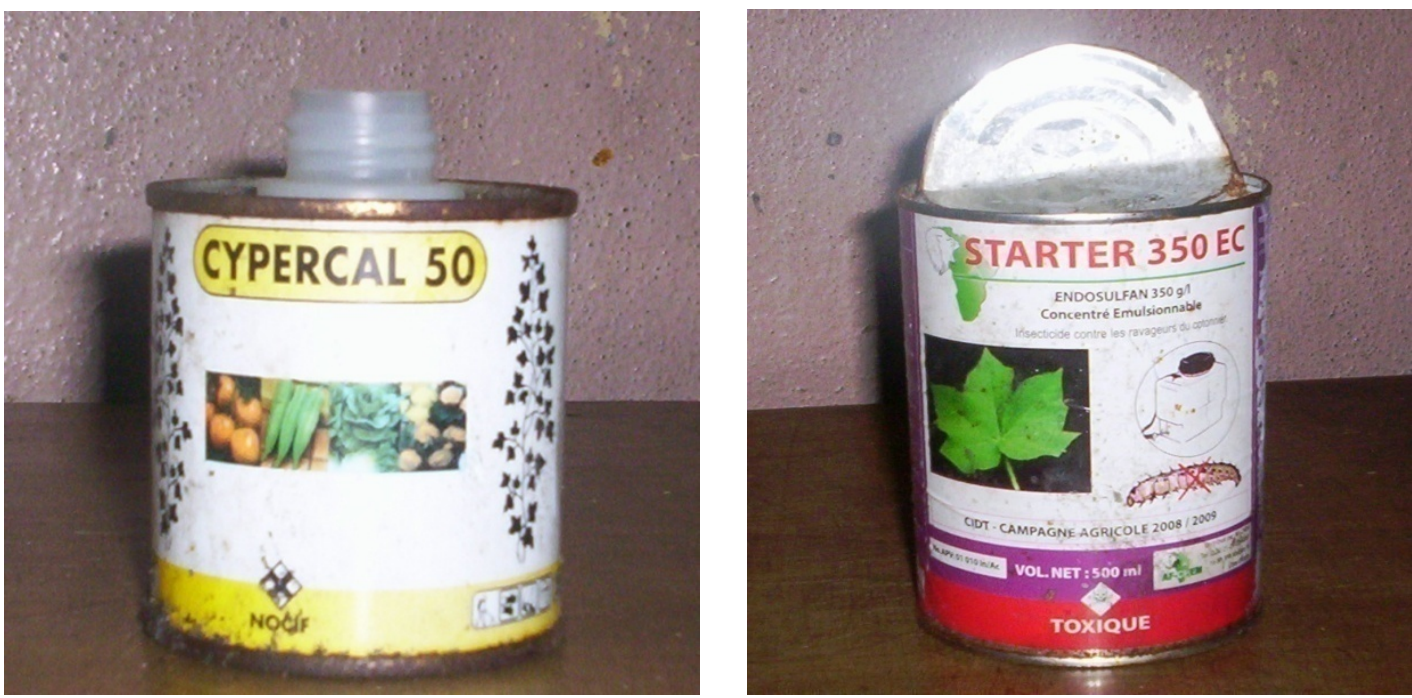

Figure 5 : Emballage de Cypercal 50

Figure 6 : Emballage de Starter 350 EC

Les informations fournies par les étiquettes à savoir le nom du produit commercial, la matière active, la quantité de matière active dans le produit commercial, le type de formulation, le degré de toxicité, la culture à laquelle le produit est destiné, le numéro d'homologation, le nom du fabricant ou du distributeur ainsi que le nombre de boîtes découvertes ont été consignées dans le tableau 2. Ces emballages appartenaient à vingt-deux (22) produits commerciaux dont treize (13) insecticides, deux (2) fongicides, cinq (5) à la fois insecticides et acaricides, et deux (2) à la fois insecticides et nématicides. Ces pesticides n'étaient pas tous homologués par l'Etat de Côte d'Ivoire. Deux produits commerciaux provenaient du Ghana. Ces deux spécialités agrochimiques répondaient aux noms de Lambda 2,5 EC et de Lambda Super 2,5 EC et étaient respectivement distribués par B. Kaakyire Agro Chemical Company et Kumark Trading Ent, basés au Ghana. Ces produits ne portaient pas de numéro d'homologation. Hormis ces deux produits agrochimiques, les autres spécialités étaient mises sur le marché par AFCHEM, Allgro, Callivoire, AGRO, ALM, STEPC/SOLEVO et Syngeta/RMG qui sont des firmes implantées en Côte d'Ivoire.

Tableau 2: Caractéristiques des produits agrochimiques utilisés par les maraîchers

\begin{tabular}{|c|c|c|c|c|c|c|}
\hline $\begin{array}{l}\text { Nom } \\
\text { Commercial }\end{array}$ & $\begin{array}{l}\text { Matière Active } \\
\text { (MA) }\end{array}$ & [MA] & NBD & $\mathbf{U} \mathbf{R}$ & $\begin{array}{l}\mathrm{N}^{\circ} \\
\text { d'Homolo. }\end{array}$ & F/D \\
\hline Almaneb $80 \mathrm{WP}$ & Manèbe & $80 \%$ & 12 & maraîcher & $960347 \mathrm{Fo}$ & ALM \\
\hline \multirow[t]{2}{*}{ Banko Plus } & Chlorothalonil & $550 \mathrm{~g} / \mathrm{L}$ & \multirow[t]{2}{*}{3} & \multirow[t]{2}{*}{ maraîcher } & \multirow[t]{2}{*}{$990483 \mathrm{Fo}$} & \multirow[t]{2}{*}{ Callivoire } \\
\hline & Carbendazime & $100 \mathrm{~g} / \mathrm{L}$ & & & & \\
\hline Calfos 720 EC & Profenofos & $720 \mathrm{~g} / \mathrm{L}$ & 1 & coton & 070759 In & Callivoire \\
\hline Callidim 40 EC & Dimethoate & $400 \mathrm{~g} / \mathrm{L}$ & 4 & boiserie & 980417 In & Callivoire \\
\hline Cigogne $50 \mathrm{EC}$ & Cypermethrine & $50 \mathrm{~g} / \mathrm{L}$ & 1 & maraîcher & 040652 In & $\begin{array}{l}\text { STEPC/ } \\
\text { SOLEVO }\end{array}$ \\
\hline Curacron 500EC & Profenofos & $500 \mathrm{~g} / \mathrm{L}$ & 6 & coton & $990466 \mathrm{In} / \mathrm{Ac}$ & Syngeta/RGM \\
\hline \multirow[t]{2}{*}{ Cypalm 336 EC } & Cypermethrine & $36 \mathrm{~g} / \mathrm{L}$ & \multirow[t]{2}{*}{1} & \multirow[b]{2}{*}{ coton } & \multirow[b]{2}{*}{990494 In } & \multirow[b]{2}{*}{ ALM } \\
\hline & Profenofos & $300 \mathrm{~g} / \mathrm{L}$ & & & & \\
\hline
\end{tabular}




\begin{tabular}{|c|c|c|c|c|c|c|}
\hline Cypalm 50 EC & Cyperméthrine & $50 \mathrm{~g} / \mathrm{L}$ & 2 & maraîcher & 000494 In & ALM \\
\hline Cypercal 50 EC & Cyperméthrine & $50 \mathrm{~g} / \mathrm{L}$ & 10 & maraîcher & $900100 \mathrm{In}$ & Callivoire \\
\hline Diafuran $5 G$ & Carbofuran & $5 \%$ & 1 & maraîcher & $010552 \mathrm{In} / \mathrm{Ne}$ & Callivoire \\
\hline \multirow[t]{2}{*}{ Duel 186 EC } & Profénofos & $150 \mathrm{~g} / \mathrm{L}$ & \multirow[t]{2}{*}{7} & \multirow[b]{2}{*}{ coton } & \multirow[b]{2}{*}{000518 In } & \multirow[b]{2}{*}{ AF-CHEM } \\
\hline & Cyperméthrine & $36 \mathrm{~g} / \mathrm{L}$ & & & & \\
\hline Furadan $5 \mathrm{G}$ & Carbofuran & $5 \mathrm{~g} / \mathrm{kg}$ & 2 & maraîcher & $\begin{array}{l}900091 \\
\mathrm{In} / \mathrm{Ne}\end{array}$ & $\begin{array}{l}\text { STEPC/ } \\
\text { SOLEVO }\end{array}$ \\
\hline Kart 500 SP & Cartap & $500 \mathrm{~g} / \mathrm{L}$ & 26 & maraîcher & $970355 \mathrm{In}$ & $\begin{array}{l}\text { STEPC/ } \\
\text { SOLEVO }\end{array}$ \\
\hline Lambad 2,5 EC & Lambdacyhalothrine & $25 \mathrm{~g} / \mathrm{L}$ & 1 & maraîcher & pas & BACC \\
\hline $\begin{array}{l}\text { Lambda Super 2,5 } \\
\text { EC }\end{array}$ & Lambdacyhalothrine & $25 \mathrm{~g} / \mathrm{L}$ & 2 & maraîcher & pas & K.T. Ent, \\
\hline Lambdax 2, 5 EC & Lambdacyhalothrine & $25 \mathrm{~g} / \mathrm{L}$ & 1 & maraîcher & Pas & AGRO \\
\hline \multirow[t]{2}{*}{ Lamdex 315 Ec } & Lambdacyhalothrine & $15 \mathrm{~g} / \mathrm{L}$ & \multirow[t]{2}{*}{2} & \multirow[b]{2}{*}{ coton } & \multirow[b]{2}{*}{060708 In } & \multirow[b]{2}{*}{ Callivoire } \\
\hline & Chlorpyrifos Ethyle & $300 \mathrm{~g} / \mathrm{L}$ & & & & \\
\hline Lampfos $168 \mathrm{Ec}$ & $\begin{array}{l}\text { Lambdacyhalothrine } \\
\text { Profénofos }\end{array}$ & $\begin{array}{l}18 \mathrm{~g} / \mathrm{L} \\
150 \mathrm{~g} / \mathrm{L}\end{array}$ & 1 & coton & 08090 In & Allgro \\
\hline \multirow[t]{2}{*}{ Polytrine186 Ec } & Cyperméthrine & $36 \mathrm{~g} / \mathrm{L}$ & \multirow[t]{2}{*}{11} & \multirow[b]{2}{*}{ coton } & \multirow[b]{2}{*}{ 950329-In/Ac } & \multirow[t]{2}{*}{ Syngeta/RGM } \\
\hline & Profénofos & $150 \mathrm{~g} / \mathrm{L}$ & & & & \\
\hline Pyrical 480 Ec & Chlorpyrifos Ethyle & $\begin{array}{l}\text { Non } \\
\text { précisée }\end{array}$ & 4 & boiserie & 000498 In & Callivoire \\
\hline Starter $350 \mathrm{Ec}$ & Endosulfan & $350 \mathrm{~g} / \mathrm{L}$ & 1 & coton & $\begin{array}{l}\text { Apv : } 01010 \\
\text { In/ Ac }\end{array}$ & AF-CHEM \\
\hline Tango $500 \mathrm{Ec}$ & Profénofos & $500 \mathrm{~g} / \mathrm{L}$ & 2 & coton & $\begin{array}{l}020575 \text { In / } \\
\text { Ac }\end{array}$ & AF-CHEM \\
\hline
\end{tabular}

[MA]: concentration de la matière active ; BACC : B KAAKYIRE AGRO CHEMICAL COMPANY; F/D : Fabricant ou Distributeur; K.T. Ent.: Kumark Trading Ent; MA : Matière Active; Numéro d'Homolo : Numéro d'homologation; NBD : Nombre de Boîtes Découvertes ; UR : Usage Recommandé ; In : insecticide ; Ac : acricide ; $\mathrm{Ne}$ : nématocide

Les produits agrochimiques étaient majoritairement des insecticides $(65,35 \%)$. Les produits à action insecticides et acaricides arrivaient en seconde position (18,81\%), suivis des fongicides $(14,85 \%)$ et des produits à la fois insecticides et nématicides $(2,87 \%)$ (figure7). 


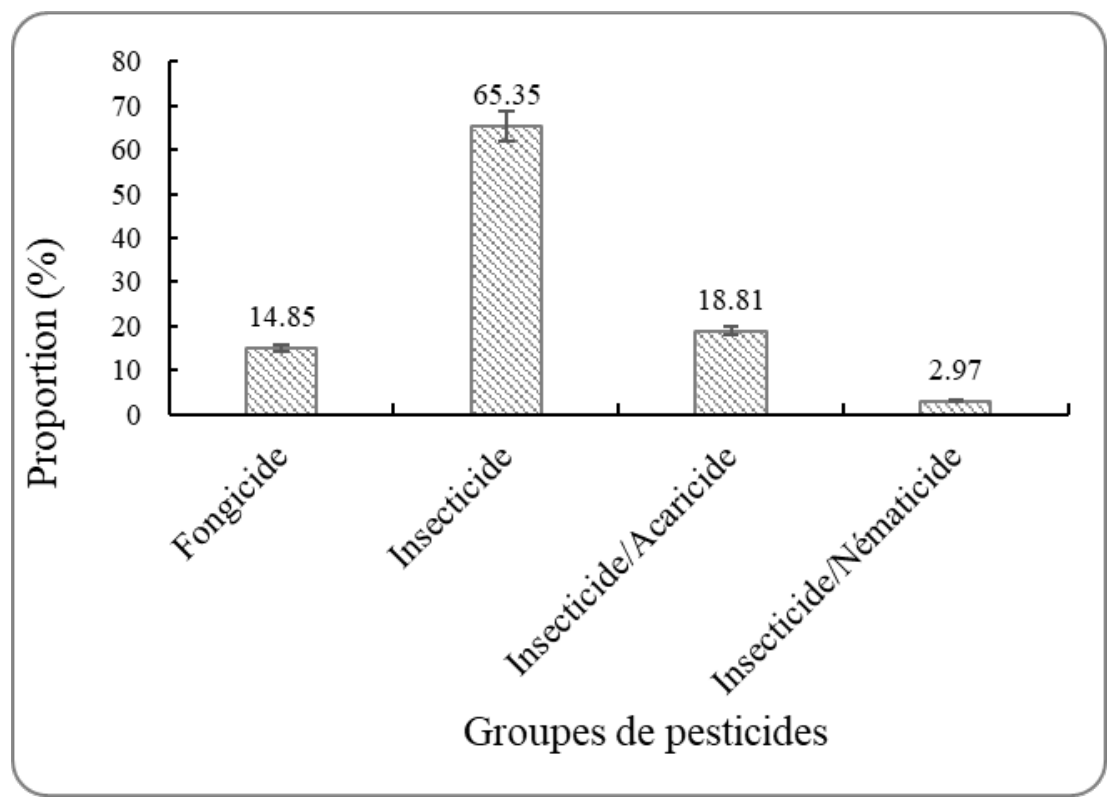

Figure 7 : Histogramme des différents groupes de pesticides

Sur le terrain, les maraîchers achetaient les produits agrochimiques avec des revendeurs ambulants et des revendeurs basés sur le site de l'ASECNA. Les produits phytosanitaires étaient empilés les uns sur les autres sous des hangars, donnant un faciès qui était loin de satisfaire les bonnes conditions de stockage des produits agrochimiques. Les produits commerciaux étaient à base de 11 matières actives qui étaient l'endosulfan, la lambdacyhalothrine, le profénofos, le chlorpyrifos éthyle, la cyperméthrine, le manèbe, le dimethoate, le cartap, le carbendazime, le chlorothalonil et le carbofuran. La cyperméthrine, le profénofos, et le cartap étaient les plus abondants avec des proportions respectives de 25,4\%, 23,02 $\%$ et $20,63 \%$ (figure 8 ).

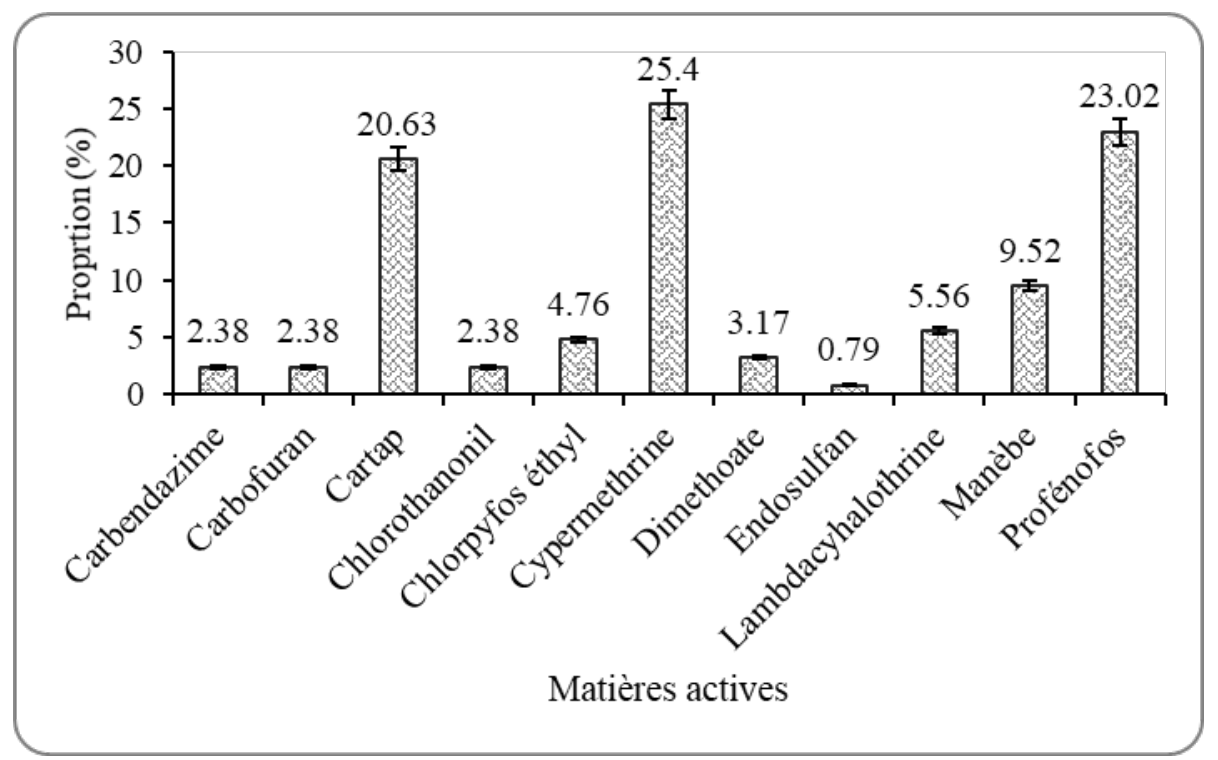

Figure 8 : Histogramme des différentes matières utilisées par les maraîchers du 43éme BIMa et de l'ASECNA

Les matières actives appartiennent à six grandes familles chimiques de pesticides à savoir les benzimidazoles, les organochlorés, les organophosphorés, les carbamates, les phthalimides et les pyréthroïdes. Les carbamates, les organophosphorés et les pyréthrinoïdes étaient les 
pesticides les plus abondants. Les carbamates étaient prépondérants avec une proportion de 32,54 $\%$. La famille des organophosphorés et celle des pyréthrinoïdes étaient en seconde position et représentaient chacune $30,95 \%$ des pesticides recensés. Les phthalimides et les benzimidazoles occupaient la quatrième place, ils représentaient chacun 2,8\% des matières actives utilisées. Les organochlorés étaient en dernière position avec une proportion de $0,79 \%$ (figure 9 ).

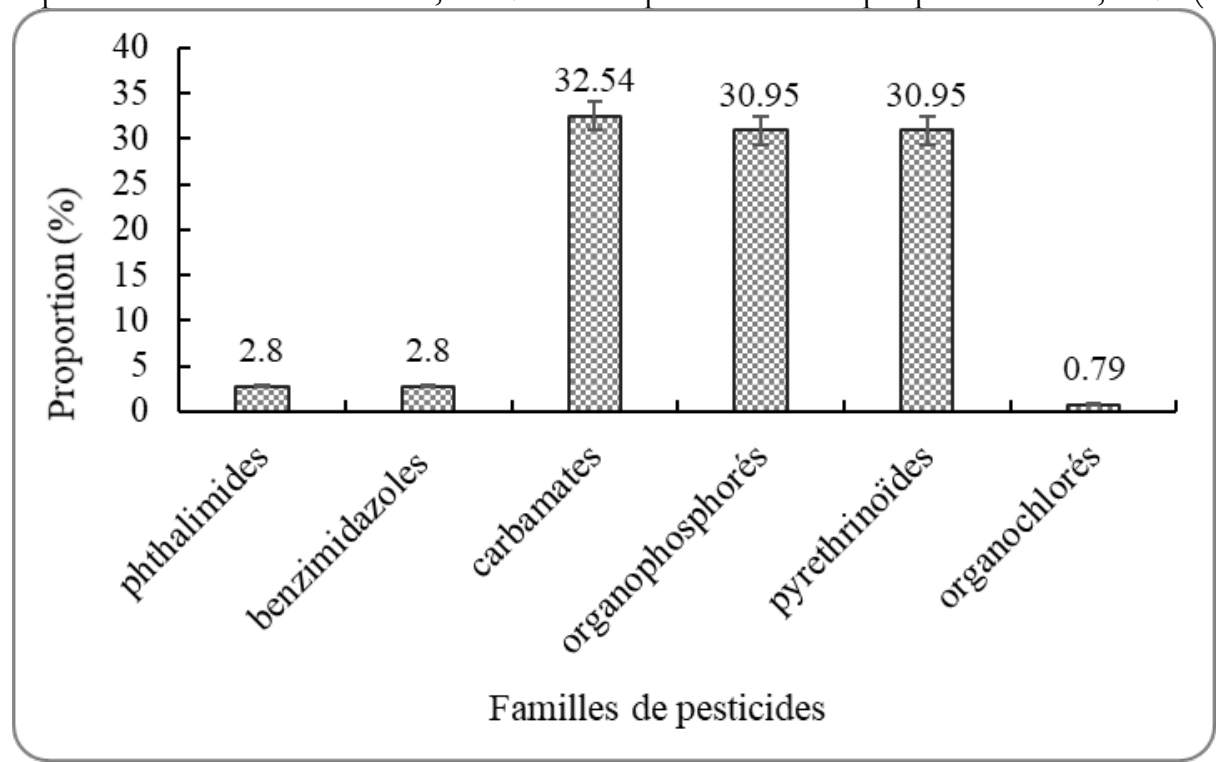

Figure 9: Histogramme des familles de pesticides utilisés par les maraîchers du 43éme BIMa et de l'ASECNA

Parmi ces produits commerciaux $60,4 \%$ étaient recommandés au maraîchage contre 39,6 \% de produits non recommandés au maraîchage. Sur les $39,6 \%$ des pesticides non recommandés à la production légumière, $80 \%$ étaient homologués pour la culture du coton tandis que $20 \%$ étaient destinés à la boiserie. Les maraîchers adoptaient deux modes d'épandage des pesticides à savoir la pulvérisation de bouillie et la dissémination des granulés. L'épandage des bouillies se faisait au moyen de pulvérisateur à dos à pression constante tandis que la dissémination des granulés s'effectuait à la volée. Lors de l'épandage des produits phytosanitaires, la majeure partie des applicateurs ne portaient pas d'équipement de protection (combinaisons, masques adéquats, gants, lunettes ou bottes), même si ces derniers évitaient de pulvériser la bouillie contre le sens du vent (figure 10).

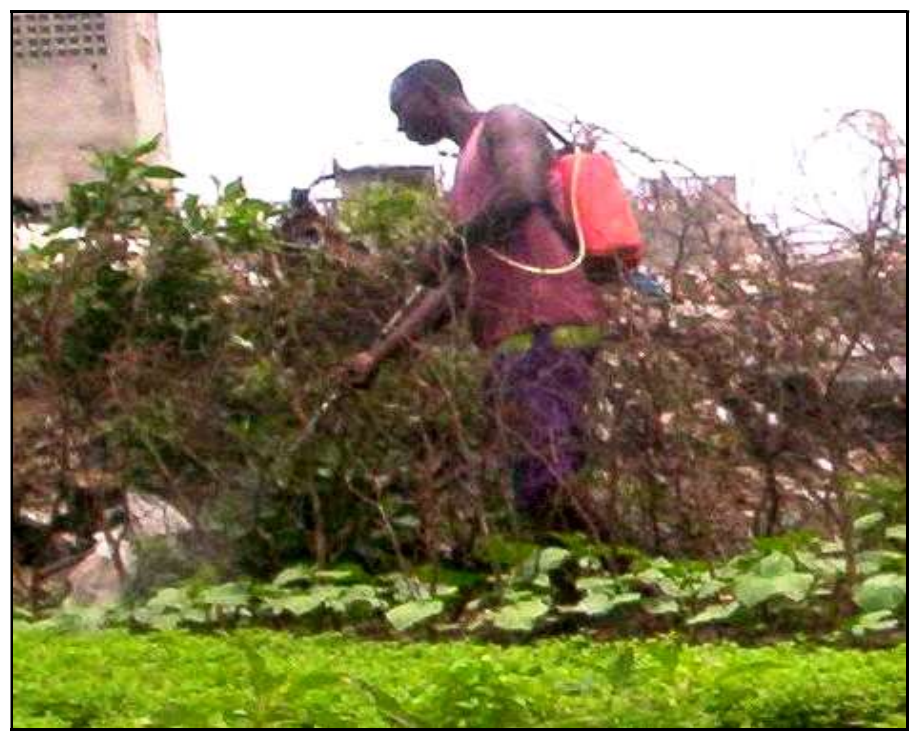


Figure 10 : Application de produit phytosanitaire sans protection (Site du 4⿳̀̀me BIMa)

La proportion des maraîchers qui avait avoué se protéger pendant l'épandage des produits phytosanitaires était de 13,16\%. A l'opposé, 86,84 $\%$ de maraîchers avaient affirmé ne pas se protéger au cours de l'application des produits agrochimiques. Les applications des produits phytosanitaires se faisaient sans opération de calibrage préalable et le mesurage des quantités de pesticides se faisait à l'aide de capsules de boissons (Coca cola, Fanta) ou de produits agrochimiques, de cuillères à soupe et parfois de dosettes adéquates. Les délais avant récolte (DAR) des produits agrochimiques dont les emballages avaient été collectés sur les sites de production, ont varié entre sept (07) et 21 jours. La comparaison de ces délais avant récolte indiqués à ceux observés par les maraîchers, avait révélé que la majeure partie de ces agriculteurs $(80,26 \%)$ récoltaient leurs légumes ou les vendaient avant le DAR. Selon les maraîchers, un arrosage abondant était suffisant pour lessiver les pesticides afin de sécuriser la santé des consommateurs. Plusieurs malaises notamment le rhume, les céphalées, la toux, les coliques et le vertige survenaient chez $75 \%$ des maraîchers après l'épandage des pesticides. Pour lutter contre les troubles provoqués par les pesticides, les applicateurs absorbaient du lait concentré non sucré (Bonnet rouge) et les antalgiques antipyrétiques tels que le paracétamol (figure 11).

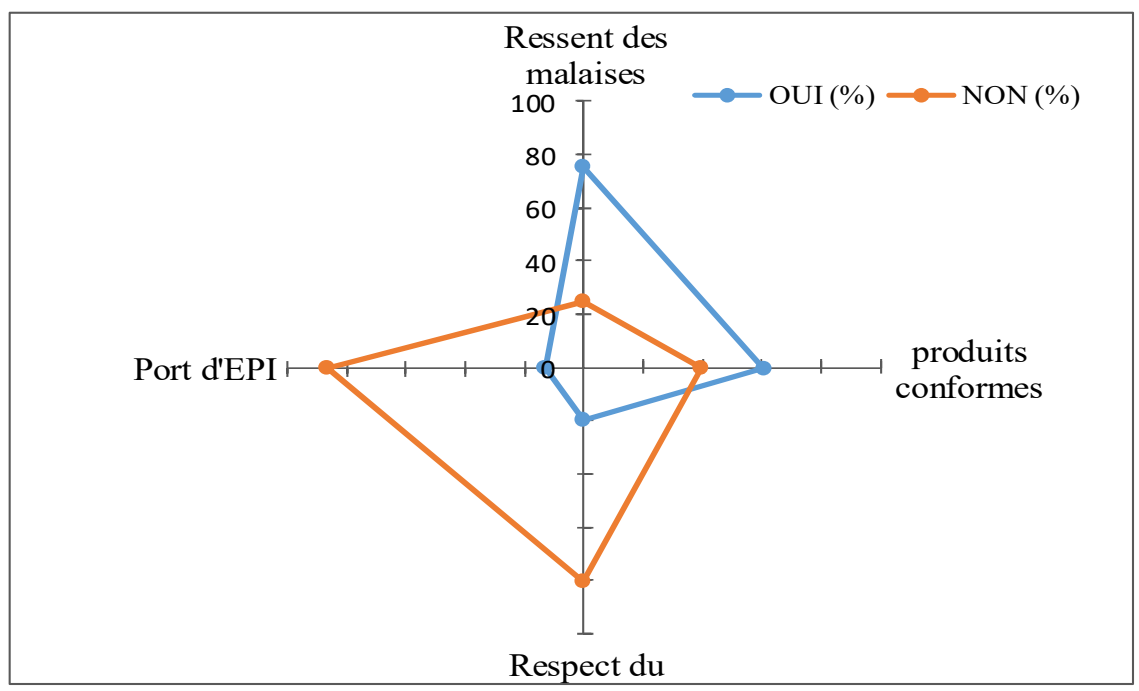

EPI : équipement de protection individuelle; DAR : délai avant récolte

Figure 11 : Radar de la distribution des maraîchers en fonction des pratiques phytosanitaires

4.6 Recherche des résidus de pesticides dans la laitue : Au total, 30 échantillons de laitue ont été analysés. Les teneurs moyennes en résidus de pesticides de ces échantillons ont été de $0,75 \pm$ $0,04 \mathrm{mg} / \mathrm{Kg}$ pour le chlorothalonil; $0,05 \pm 0,02$ $\mathrm{mg} / \mathrm{Kg}$ pour le manèbe ; $0,07 \pm 0,03 \mathrm{mg} / \mathrm{Kg}$ pour le chlorpyrifos éthyle; $0,14 \pm 0,06 \mathrm{mg} / \mathrm{Kg}$ pour le carbendazime; $0,97 \pm 0,42 \mathrm{mg} / \mathrm{Kg}$ pour la cyperméthrine; $7,83 \pm 1,72 \mathrm{mg} / \mathrm{Kg}$ pour le lambdacyhalothrine et $0,92 \pm 0,43 \mathrm{mg} / \mathrm{Kg}$ pour le dimethoate. Les limites maximales de résidus auxquelles ces teneurs avaient été comparées étaient celles du Codex alimentarius et de l'Union Européenne (tableau 3).

Tableau 3 : Teneurs moyennes en résidus de pesticides de la laitue comparées aux LMR

\begin{tabular}{l|c|c|c|l}
\hline Matières actives & $\begin{array}{c}\text { Teneur } \\
(\mathbf{m g} / \mathbf{K g})\end{array}$ & & $\begin{array}{c}\mathbf{L M R} \\
\mathbf{( m g} / \mathbf{K g})\end{array}$ & Organisme/année \\
\hline Chlorothalonil & $0,05 \pm 0,02$ & $<$ & 0,3 & Codex alimentarius $/ 2011$ \\
Manèbe & $0,07 \pm 0,03$ & $<$ & 5 & Codex alimentarius $/ 2008$ \\
Chlorpyrifos éthyle & $0,14 \pm 0,06$ & $>$ & 0,01 & Reg. UE 2018/686 \\
\hline
\end{tabular}




\begin{tabular}{l|c|c|c|l}
\hline Carbendazime & $0,97 \pm 0,42$ & $<$ & 5 & Codex alimentarius /2009 \\
Cyperméthrine & $0,97 \pm 0,42$ & $>$ & 0,7 & Codex alimentarius/ 2009 \\
Lambdacyhalothrine & $7,83 \pm 1,72$ & $>$ & 0,3 & Codex alimentarius /2009 \\
Dimethoate & $0,92 \pm 0,43$ & $>$ & 0,3 & Codex alimentarius /2009 \\
\hline
\end{tabular}

Reg UE. : Règlement de l'Union Européenne

Au regard des teneurs en résidus de pesticides et des normes du codex alimentarius et de l'Union Européenne, cinq (05) matières actives s'étaient distinguées par rapport à leurs proportions d'échantillons non conformes (ENC) ou impropres à la consommation. Il s'agissait du lambdacyhalothrine (93,33\% ENC), du dimethoate (36,67 $\%$ ENC), de la cyperméthrine $(26,27 \%$ ENC), du chlorpyrifos $(23,33 \% \mathrm{ENC})$ et du chlorothalonil (10\% ENC) (figure 12).

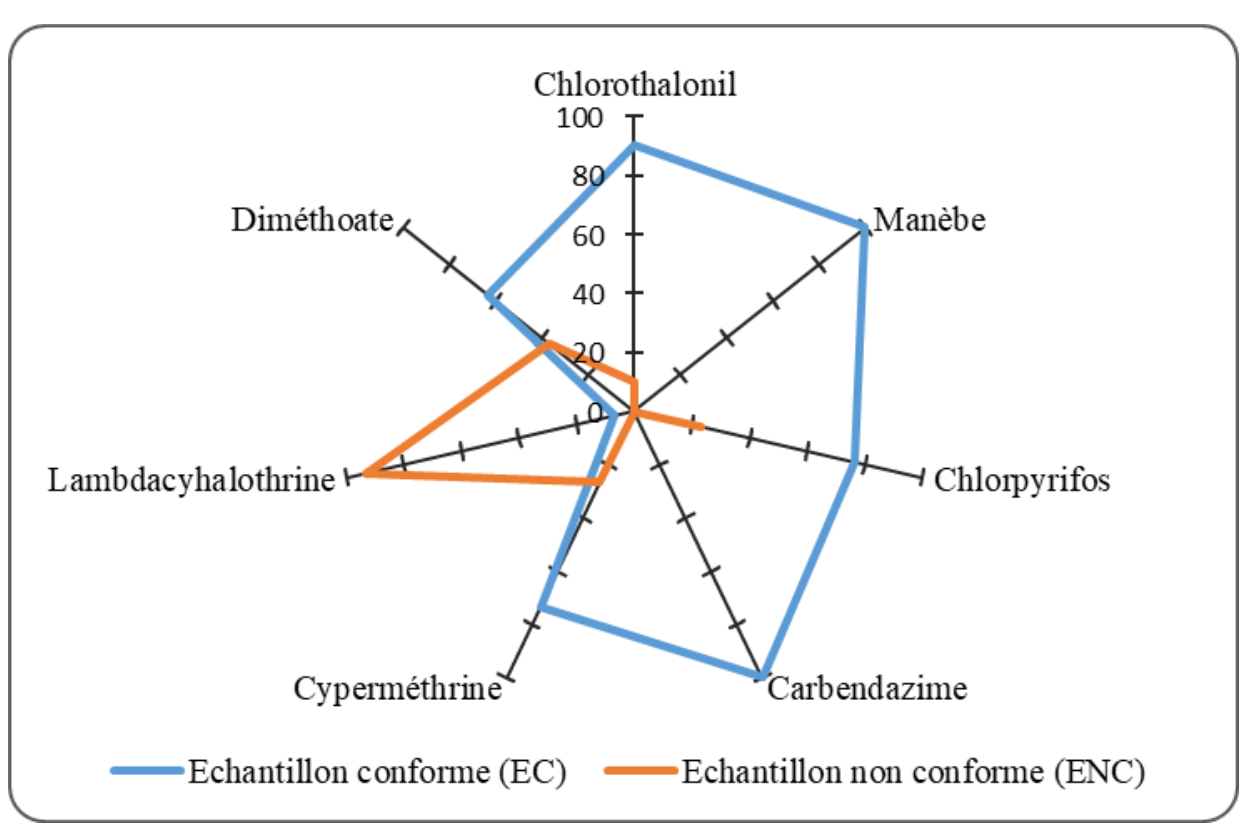

Figure 12 : Radar de la conformité des échantillons aux LMRs en fonction des matières actives

En somme, la prise en compte simultanée de tous les pesticides a conduit à 93,33\% des échantillons de laitue non conformes aux LMRs du Codex alimentarius et de l'UE (Figure 13). 


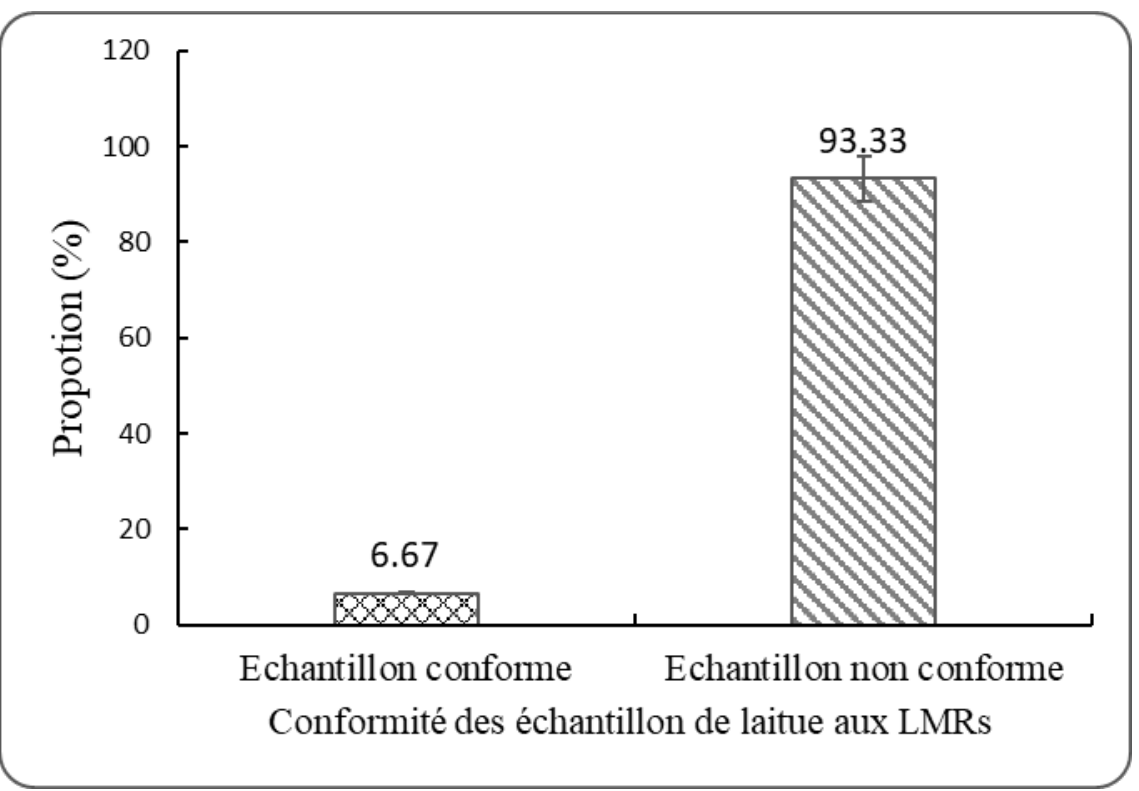

Figure 13 : Histogramme de conformité des échantillons de laitue aux LMRs

\section{DISCUSSION}

La présente étude a montré que la production de maraîchers à Port-Bouët était largement dominée par les hommes qui représentaient 98,68\% des producteurs. Les femmes étaient très minoritaires avec une proportion de $1,32 \%$. Les femmes s'investissaient plutôt dans la commercialisation des produits maraîchers. Elles récoltaient ou collectaient les légumes à partir des sites de production et les acheminaient vers les marchés ou vers les quartiers pour les commercialiser ; cela pourrait expliquer la faible implication de la gente féminine dans la mise en place des cultures. Ces résultats confortent ceux de Kouakou et al.(2010), qui avaient montré que les femmes représentaient seulement $6 \%$ des maraîchers de la commune de Yopougon (Abidjan). Le faible taux de scolarisation des maraîchers et leur bas niveau d'étude pourraient s'expliquer par le taux de pauvreté qui reste élevé dans la ville d'Abidjan et le fait que l'école n'était pas obligatoire en son temps. Selon l'enquête niveau de vie de ménages (INS, 2015), la ville d'Abidjan est celle qui contribue le plus à la pauvreté en milieu urbain (9,3\%). Aussi, avec le manque d'emploi, n'est-il pas évident pour des individus ayant un niveau d'étude inférieur ou égal CEPE de postuler pour les offres. La sélection est rude et les non diplômés sont contraints d'opter pour une occupation qui ne leur impose pas de diplôme. Le maraîchage urbain est parmi tant d'autres activités du secteur informel, un point de chute pour ces chercheurs d'emploi non qualifiés. C'est la raison pour laquelle, beaucoup de maraîchers qualifient ce travail de temporaire ou transitoire. Ainsi, quelques producteurs de plantes maraîchères exercent cette activité en jumelage avec le commerce, la maçonnerie, la menuiserie, la charpenterie, la briqueterie ou l'artisanat d'art (vannerie, broderie, couture etc.) (N’Diénor et Aubry, 2004). Le faible taux de scolarisation des maraîchers et le bas niveau d'étude de ces agriculteurs sont des faits que Kanda et al. (2009) ont aussi signalés chez les maraîchers de Lomé. Le non-respect des bonnes pratiques agricoles pourrait s'expliquer d'une part par le faible taux de scolarisation, le bas niveau d'instruction des producteurs et le manque de formation qualifiante. Seulement 17,11 \% des producteurs étaient scolarisés. De plus ceux qui étaient scolarisés, avaient un bas niveau d'étude : $15,79 \%$ avaient un niveau d'études $\leq$ au Certificat d'Etude Primaire Elémentaire (CEPE) et 1,32\% avaient un niveau d'études compris entre le CEPE et le baccalauréat. En outre, ces maraîchers exerçaient à 94,74 \% dans ce secteur d'activité sans formation adéquate. Dans de telles conditions, la probabilité de bien connaître les pesticides et leur écotoxicité était infime et l'ignorance des dangers chroniques pourrait entretenir l'esprit de ces agriculteurs urbains. Leur attention était plutôt attirée par la toxicité aiguë des 
produits phytosanitaires car celle-ci était directe. Toutes les précautions prises visaient donc à éviter les effets directs (toxicité aiguë) des pesticides. Une fois ces effets évités, les maraîchers se sentaient en parfaite sécurité ignorant qu'ils manipulaient des produits que l'on peut qualifier de «bombe à retardement» à causes de leurs effets tardifs. Le faible pouvoir d'achat des maraîchers dans leur grande majorité pourrait jouer un grand rôle dans le non-respect de la bonne pratique agricole. En effet, les maraîchers qui, en dépit de leur faible niveau d'études avaient connaissance des effets à long terme des produits agrochimiques, ont avoué ne pas disposer de moyen financier pour l'acquisition de matériel adéquat de protection. Cette même situation économique pourrait aussi être à la base de la ruée de ces producteurs vers les produits agrochimiques moins coûteux, mais déconseillés pour la production légumière. C'est le cas des spécialités agrochimiques comme Stater 350 EC, Calfos 720 EC, Curacron 500 EC, Polytrine 186 EC, qui étaient homologués pour la culture du coton, mais qui ont été malheureusement utilisées par les maraîchers de Port - Bouët. Cet acte a été qualifié de détournement de produits

\section{CONCLUSION}

Les pratiques agricoles observées par les producteurs de cultures maraîchères de la commune de Port-Bouët ne respectent pas les bonnes pratiques phytosanitaires. Les délais avant récolte (DAR) des produits agrochimiques ne sont pas respectés par les maraîchers. Une bonne part des spécialités agrochimiques utilisées n'est pas adaptée aux cultures maraîchères. Ces agriculteurs assurent la production des fruits et légumes au prix de leur santé car ils appliquent les pesticides sans se protéger convenablement. L'ensemble des pratiques phytosanitaires observées par les maraîchers de la ceinture du 43 ${ }^{\text {ème }}$ BIMa et ceux qui opèrent dans la

\section{REFERENCES}

Adiko AF, Matthys B, Cissé G, Wyss K, Tschannen AB, Tanner M. et Utzinger J : 2006. Le réseau social des maraîchers à Abidjan agit sur la perception des préoccupations et des risques sanitaires liés à l'eau. Vertigo hors série $3: 11 \mathrm{p}$.

Ambrus A, Lantos J, Visi E, Csatlos I. et Sarvari L: 1981. Journal of Association of Official Analytical Chemists 64: 733p. phytosanitaires par Doumbia et Kwadjo (2009), au cours de leurs travaux sur les pratiques d'utilisation et de gestion des pesticides par les maraîchers de la ville d'Abidjan et deux de ses banlieues (Dabou et Anyama). En fait, ces produits étaient vendus à bas prix grâce à la subvention dont bénéficiait la culture du coton. La même raison financière pourrait également jouer un rôle prépondérant dans le nonrespect des DAR. En effet, même si le maraîcher est conscient des dangers chroniques potentiels des pesticides, son intérêt immédiat est le gain d'argent. Plus vite il vend sa production, plus il engrangerait de l'argent, car les DAR assez longs, pouvaient conduire à la perte des cultures ou à l'usage supplémentaire de pesticides pour lutter contre d'autres attaques des ravageurs ou maladies; ce qui fait appel à des dépenses additionnelles. Ce nonrespect du DAR par les maraîchers pourrait expliquer le fait que $93,33 \%$ des échantillons de laitue étaient non conformes aux normes du Codex ou de l'UE. En effet, le non-respect du DAR ne donne pas de temps suffisant aux matières actives et aux adjuvants de se dégrader complètement ou de s'estomper convenablement pour atteindre les teneurs non nuisibles à la santé.

zone de l'ASECNA, condusent à la non-conformité des échantillons de laitue aux limites maximales de résidus de pesticides en vigueur. Par conséquent, la consommation des fruits et légumes produits par ces agriculteurs urbains pourrait conduire à la manifestation des effets aigus et chroniques liés aux produits agrochimiques au sein de la population. Pour remédier à cette situation, des mesures d'accompagnements tels que les fonds de financement, la formation et le suivi doivent être appliquées aux maraîchers de la commune de PortBouët.

De Jaeger C, Voronska E, Fraoucene N. et Cherin P : 2012. Exposition chronique aux pesticides, santé et longévité. Rôle de notre alimentation. Médecine \& Longévité $4: 75$ 92.

Dembélé A, Oumarou B, Traore S K., Mamadou K, Coulibaly DT. et Abba T: 2008. The chemical control of the pests in the truck farming and the quality of vegetables in 
african urban cities: the health hazards and security of consumers. European Journal of Scientific Research 20(4):836-843.

Dembélé A, Oumarou B. et Abba T : 2011. Quality of vegetables and pests control in african urban cities, pesticides in the modern world - risks and benefits. InTech $32 \mathrm{p}$.

Doumbia M. et Kwadjo KE: 2009. Pratiques d'utilisation et de gestion des pesticides par les maraîchers en Côte d'Ivoire : Cas de la ville d'Abidjan et deux de ses banlieues (Dabou et Anyama). Journal of Applied Biosciences 18:992-1002.

FAO/OMC : 2018. Le commerce et les normes alimentaires. FAO et OMC 72p.

INS: 2015 : Enquête niveau de vie des nénages en Côte d'Ivoire. 91p.

Jouve AM. et Padilla M : 2007. Les agricultures périurbaines méditerranéennes à l'épreuve de la multifonctionnalité : comment fournir aux villes une nourriture et des paysages de qualité ? Cahiers Agricultures 16(4): 311317.

Kanda M, Wala K, Batawila K, Ahanchede A. et Akpagana K: 2009. Le maraîchage urbain à Lomé, risques sanitaires et dynamiques spatiales. Cahier Agriculture 18(4): 356-363.

Koffi-Nevry R, Assi-Clair BJ, Assemand EF, Affou SW. et Koussemon M : 2012. Origine des témoins de contamination fécale de l'eau d'arrosage de la laitue (Lactuca sativa) cultivée dans la zone périurbaine d'Abidjan. Journal of Applied Biosciences $52: 3669$ 3675.

Kouakou YE, Koné B, Bonfoh B, Kientga SM, N'Go YA, Savane I. et Cissé G : 2010. L'étalement urbain au péril des activités agro-pastorales à Abidjan. Vertigo 10 (2): 9p.

Koutros S, Lynch CF, Xiaomei M, Lee WJ, Hoppin JA. Christensen CH, Andreotti G, Freeman LB, Rusiecki JA, Lifang H, Sandler DP. et Alavanja MCR: 2009. Aromatic amine pesticide use and human cancer risk: results from the U.S. Agricultural Health Study. International Journal of Cancer 5(124):1206 $-1212$.

Kpan Kpan KG, Ehouman NM, Touré M, Yao B.L., Tiho S, Traoré KS. et Dembélé A : 2018. Toxicité d'un herbicide à base de glyphosate sur le ver de terre Eudrilus Eugeniae KINBERG, 1867 (Oligochaeta,
Eudrilidae). European Journal of Scientific Research 150(3) : 285-298.

Kpan Kpan GK, Tiho S, Ehouman MN, Touré M. et Dembélé A : 2017. Évaluation de la toxicité d'un herbicide à base de 2,4-D sur le ver de terre Eudrilus engeniae Kinberg, 1867 (Oligochaeta, Eudrilidae). Revue Marocaine des Sciences Agronomique et Vétérinaire 5 (3):304-312.

Meeker JD, Barr DB. et Hauser R: 2008. Human semen quality and sperm DNA damage in relation to urinary metabolites of pyrethroid insecticides. Human Reproduction 23(8): 1932-1940.

Michelin : 1998. Michelin 57 Côte d'ivoire. Michelin Services de Tourisme, 46, av. de Breteuil 75324 Paris Cedex 07.

Multigner L: 2005. Effets retardés des pesticides sur la santé humaine. Environnement, Risques \& Santé 4(3):187-194.

N'Diénor M. et Aubry C: 2004. Diversité et flexibilité des systèmes de production maraîchères dans l'agglomération d'Antananarivo (Madagascar) : atouts et contraintes de la proximité urbaine. Cahiers Agricultures 13: 50-57.

Olahan A : 2010. Agriculture urbaine et stratégies de survie des ménages pauvres dans le complexe spatial du district d'Abidjan. Vertigo 10(2): 24 p.

OMS/FAO : 2010 : Code international de conduite pour la :distribution et l'utilisation des pesticides : Directives pour l'homologation des pesticides. OMS et FAO 43p.

Regelions RV, Guadalupe OG, Victor HBA, Javier M. et Mariano ECG: 2007. Organophosphorus pesticide exposure decreases sperm quality: association between sperm parameters and urinary pesticide level. Journal of applied toxicology 28: 674-680.

Roche BS. et Ramade F : 2011. Assessment of the pesticides pollution of coral reefs communities from french polynesia. Revue Ecolgie 66: 3-10.

Sanborn M, Cole D, Kerr K, Vakil C, Sanin LH. et Bassil K : 2004. Pesticides literature review. Ontario College of family physicians. 186 p.

Tano BF, Abo K, Dembélé A. et Fondio L: 2011. Systèmes de production et pratiques à risque en agriculture urbaine: cas du 
maraîchage dans la ville de Yamoussoukro en Côte d'Ivoire. International Journal of Biological et Chemical Sciences 5(6): 23172329.

Thiam A. et Sagna MB: 2009. Monitoring des pesticides au niveau des communautés à la base. PAN Africa, $57 \mathrm{p}$.

Traoré SK, Dembélé A, Mamadou K, Mambo V, Lafrance P, Bekro YA. et Houenou P : 2008. Contrôle des pesticides organochlorés dans le lait et produits laitiers : Bioaccumulation et risques d'exposition. Afrique Science 04(1):87- 98.

Wyrobek AJ, Watchmaker G, Gordon L, Wong K, Moore D. et Whortont D: 1981. Sperm shape abnormalities in carbaryl-exposed employees. Environmental health perspectives, 40:255-265.

www.codexalimentarius.org/normes-officielles/fr/. Consulté, le 09/01/2018.

www.ec.europa.eu/food/plant/pesticides/eupesticides-database/public. Consulté, le 09/01/19.

Yao BL, Kpan Kpan KG, Messoum FG, Dembélé A. et Traoré KS: 2016. Évaluation du risque phytosanitaire lié à la consommation de la laitue (Lactuca sativa) cultivée dans la commune de Port-Bouët (Abidjan). Revue Marocaine des Sciences Agronomiques et Vétérinaire 4(3): 22-30. 